\title{
Efficacy, acceptability, and safety of adjunctive aripiprazole in treatment-resistant depression: a meta-analysis of randomized controlled trials
}

This article was published in the following Dove Press journal: Neuropsychiatric Disease and Treatment

\author{
Shuxin Luan ${ }^{1,2}$ \\ Hongquan Wan ${ }^{2}$ \\ Lei Zhang ${ }^{3}$ \\ Hua Zhao ${ }^{1,4}$ \\ 'Department of Physiology, College of \\ Basic Medical Sciences, Jilin University, \\ Changchun, China; ${ }^{2}$ Department of \\ Mental Health, The First Hospital of \\ Jilin University, Changchun, China; \\ ${ }^{3}$ Department of Radiology, The \\ First Hospital of Jilin University, \\ Changchun, China; ${ }^{4}$ Neuroscience \\ Research Center, The First Hospital of \\ Jilin University, Changchun, China
}

Background: Treatment-resistant depression (TRD) is common and potentially life-threatening in adults, and the benefits and risks of adjunctive aripiprazole in these patients remain controversial. Therefore, we conducted a meta-analysis of randomized controlled trials (RCTs) to assess the efficacy, acceptability, safety, and quality of life of adjunctive aripiprazole in patients with TRD.

Methods: RCTs published in PubMed, Web of Science, and Embase were systematically reviewed to evaluate the efficacy and safety profiles of TRD patients who were treated with adjunctive aripiprazole. The main outcome measures included response rate, remission rate, changes from baseline in Montgomery-Asberg Depression Rating Scale (MADRS), Clinical Global Impression-severity (CGI-S), Clinical Global Impression-improvement (CGI-I), 17-Item Hamilton Rating Scale for Depression (HAM-D17), Sheehan Disability scale (SDS), and Inventory of Depressive Symptomatology Self-Report Scale (IDS-SR), discontinuation due to adverse events, and adverse events. Risk ratio (RR) or weight mean difference with 95\% confidence intervals (CIs) were pooled using a fixed-effects or random-effects model according to the heterogeneity among studies.

Results: A total of 8 RCTs involving 2,260 patients were included in this meta-analysis. Adjunctive aripiprazole was associated with a significantly higher remission rate $(\mathrm{RR}=1.64,95 \% \mathrm{CI}$ : 1.42 to $1.89 ; P<0.001)$ and response rate $(\mathrm{RR}=1.45,95 \% \mathrm{CI}: 1.13$ to $1.87 ; P=0.004)$ than other treatments. Moreover, adjunctive aripiprazole had greater changes in MADRS score, CGI-S score, CGI-I score, HAM-D17 score, SDS score, and IDS-SR score. There were more patients treated with adjunctive aripiprazole who discontinued their treatments due to adverse events. The incidence of adverse events was significantly higher in the adjunctive aripiprazole group than in other treatment groups.

Conclusion: The adjunctive aripiprazole showed benefits in improving the response rate, remission rate, and the quality of life in patients with TRD. However, clinicians should interpret these findings with caution due to the evidence of potential treatment-related side effects.

Keywords: treatment-resistant depression, adjunctive aripiprazole, meta-analysis

\section{Introduction}

Major depressive disorder (MDD) is common in adults, which leads to disability, suicidality, and increased mortality. ${ }^{1-3}$ Although several available treatments have been applied for MDD over the past 2 decades, it is still a challenging illness that psychiatrists face. ${ }^{3}$ Up to $50 \%-60 \%$ of patients do not achieve adequate response, ${ }^{4}$ and two-thirds of them do not experience a timely remission. ${ }^{5}$ This is of significant concern, since patients with partial response or residual symptoms have reduced functioning and a worse 
prognosis than these with remission. ${ }^{4}$ In addition, patients who fail to achieve remission from MDD are more likely to have functional impairment and suicide. ${ }^{6}$ These patients who did not respond adequately to the conventional antidepressant therapy are broadly defined as having treatment-resistant depression (TRD). ${ }^{4}$ It is difficult to estimate the prevalence of TRD, but patients with TRD usually have poor long-term outcomes and increased risk of recurrence. ${ }^{7}$ Therefore, there is need for additional treatment strategies for those patients with TRD.

A range of augmentation and combination strategies has been used to improve the response rate and remission rate in patients with inadequate antidepressant response. ${ }^{8}$ These treatment options include mirtazapine, bupropion, and augmentation with lithium, second-generation antipsychotics, olanzapine/fluoxetine combination. ${ }^{9,10}$ Augmentation strategies involve the addition of a nonstandard agent to the treatment regimen. ${ }^{11}$ One advantage of augmentation is that it eliminates the transition period between one antidepressant to another, thereby building on any partial response (20\%-50\% improvement). ${ }^{12}$

Aripiprazole is a second atypical antipsychotic approved by the Food and Drug Administration for augmentation treatment of MDD. It is distinct from other antipsychotics acting as a partial agonist at dopamine D2, D3, and serotonin 5- $\mathrm{HT}_{1 \mathrm{~A}}$ receptors and as an antagonist at 5- $\mathrm{HT}_{2 \mathrm{~A}}$ receptors. ${ }^{13-15}$ In one 6-week prospective open-label multicenter study, adjunctive aripiprazole significantly reduced the Montgomery-Asberg Depression Rating Scale (MADRS) score by 14.0 points in patients with MDD who had inadequate antidepressant response. ${ }^{16}$ Moreover, it also improved the response rate and remission rate by $52.3 \%$ and $39.8 \%$, respectively. ${ }^{16}$ There are several clinical trials that assessed the efficacy and safety of adjunctive aripiprazole in TRD patients; however, their results remain inconsistent. Thus, we conducted this meta-analysis of randomized controlled trials (RCTs) to evaluate the efficacy, acceptability, safety, and quality of life of adjunctive aripiprazole in the treatment of patients with TRD.

\section{Methods}

\section{Search strategy}

We conducted this meta-analysis in accordance with the Preferred Reporting Items for Systematic Review and Metaanalysis criteria. ${ }^{17}$ A comprehensive search was conducted to identify relevant studies on the use of adjunctive aripiprazole in the treatment of TRD. PubMed, Embase, and Web of Science were searched for all studies published before
October 7, 2017. The search was limited to human subjects and no language restriction was imposed. The search terms used were ("depressive disorder, treatment-resistant" [MeSH Terms]) OR ("depressive" [All Fields] AND "disorder" [All Fields] AND “treatment-resistant" [All Fields]) OR "(treatment-resistant depressive disorder" [All Fields]) OR ("treatment" [All Fields] AND "resistant" [All Fields] AND "depression" [All Fields]) OR ("treatment resistant depression" [All Fields]) AND ("aripiprazole" [MeSH Terms] OR "aripiprazole" [All Fields]). In addition, we also searched the reference lists of the included studies to identify other potentially eligible studies that we may have left out with our primary search.

\section{Study selection}

All clinical trials that were assessed for the efficacy and safety of adjunctive aripiprazole for TRD were considered eligible for analysis. The selection criteria applied were as follows: 1) study design: RCT; 2) population: adult patients diagnosed with TRD; 3 ) intervention: adjunctive aripiprazole; 4) comparison intervention: any type of control; 5) outcome measure: response rate, remission rate, mean change from baseline in MADRS, Clinical Global Impression-severity (CGI-S), Clinical Global Impression-improvement (CGI-I), 17-Item Hamilton Rating Scale for Depression (HAM-D17), Sheehan Disability scale (SDS), Inventory of Depressive Symptomatology Self-Report Scale (IDS-SR), discontinuations due to adverse events, and incidence of treatmentrelated adverse events.

\section{Data extraction}

Data were extracted from selected studies independently by 2 investigators (SL and HW) using a standardized data extraction method. We extracted the following data: first author, year of publication, number of patients in each group, baseline patient characteristics, and outcomes, including remission rate, response rate, mean change from baseline in MADRS, CGI-S, CGI-I, HAM-D17, SDS, IDS-SR, and treatment-related adverse events. The data were entered into a standardized Excel file and checked by a third investigator. Any disagreements between the 2 investigators were resolved by discussion and consensus.

\section{Risk of bias and evidence grade assessment}

We used the Cochrane risk-of-bias tool to assess the risk of bias of the included study. ${ }^{18}$ Each study was assigned a value of low, unclear, or high risk of bias according to the 
following domains: random sequence generation; allocation concealment; blinding of participants and personnel to the study protocol; blinding of outcome assessment; incomplete outcome data; selective reporting; and other bias. ${ }^{18} \mathrm{We}$ also evaluated the quality of evidence for the outcome measures using the Grading of Recommendations Assessment, Development, and Evaluation (GRADE). ${ }^{19}$ Each outcome was classified as very low, low, moderate, or high quality of evidence. A summary table was prepared using the GRADE profiler (GRADEpro, version 3.6).

\section{Statistical analysis}

We estimated the risk ratio (RR) with $95 \%$ confidence intervals (CIs) for dichotomous outcomes, and weighted mean difference (WMD) with 95\% CIs for continuous outcomes. We first tested the heterogeneity between the studies using $I^{2}$ statistics, in which $I^{2}>50 \%$ indicated significant heterogeneity. ${ }^{20}$ Whenever heterogeneity was found among the included studies, a random-effects model ${ }^{21}$ was used to pool the estimates; otherwise, a fixed-effects model ${ }^{22}$ was applied. We also conducted sensitivity analysis, subgroup analysis, and meta-regression to explore the potential sources of heterogeneity whenever significant heterogeneity was present. Publication bias was assessed by the Begg and Mazumdar ${ }^{23}$ and Egger et $\mathrm{al}^{24}$ test. A $P$-value $<0.05$ was judged as statistically significant, except where otherwise specified. All statistical analyses were performed using STATA, version 12.0 (Stata Corporation, College Station, TX, USA).

\section{Results}

\section{Identification of eligible studies}

The initial search yielded 835 relevant publications, of which 416 were excluded because of duplicate studies. After reviewing the abstract and title, 406 were excluded because of various reasons (reviews, non-RCTs, or not relevant to our topics). Then 13 potentially relevant studies were identified for the full-text information analysis, and 5 were excluded because they were single-arm studies, ${ }^{16,25,26}$ or did not provide available data, ${ }^{27}$ or assigned aripiprazole in both groups. ${ }^{28}$ Finally, $8 \mathrm{RCTs}^{29-36}$ met the inclusion criteria and were included in this meta-analysis. The search flow chart is shown in Figure 1.

\section{Characteristics of eligible studies}

The main patient characteristics of the 8 included studies are presented in Table 1. All the included studies were wellperformed, prospective RCTs. Clinical characteristics were matched for age, gender, and duration of current episode in

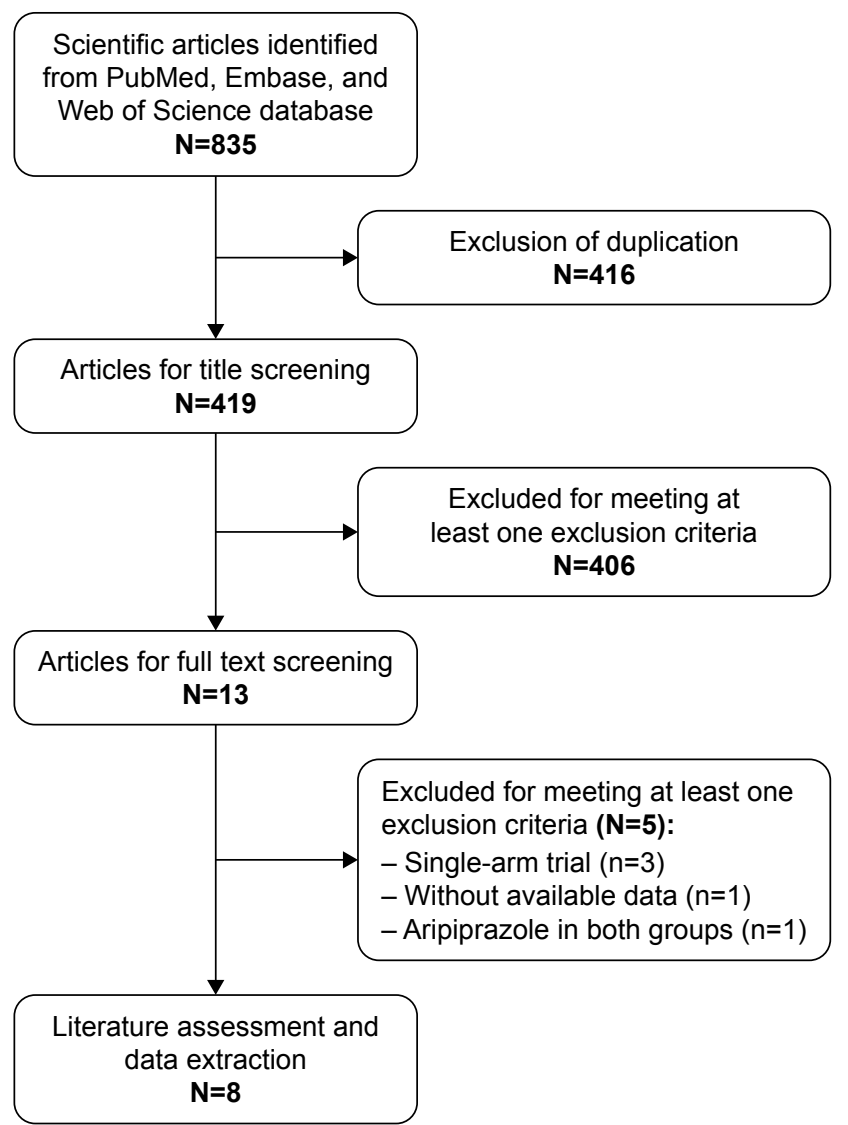

Figure I Eligibility of studies for inclusion in meta-analysis.

each study. These studies were published between 2007 and 2016. Most of patients in these studies were white, black, and Asian patients. Among the 8 trials, 5 were conducted in USA, ${ }^{29,30,32-34} 1$ in China, ${ }^{31} 1$ in Germany, ${ }^{35}$ and 1 in Japan. ${ }^{36}$ Of the included studies, 7 compared adjunctive aripiprazole with placebo, ${ }^{29-34,36}$ whereas the remaining 1 compared aripiprazole plus mirtazapine with mirtazapine monotherapy. ${ }^{35}$ The dosage of aripiprazole ranged from 2 to $20 \mathrm{mg} /$ day. The duration of follow-up ranged from 4 to 12 weeks, and most of the studies had a follow-up of 6 weeks.

\section{Risk of bias and evidence of grade assessment}

The overview of the risk of bias is summarized in Figure 2. Overall, 5 studies were classified as being at low risk of bias, 2 being at unclear risk of bias, and 1 being at high risk of bias. The reason for high risk of bias was that this study was not conducted in a double-bind design, and participants and personnel were aware of the therapeutic schedule..$^{35}$ The reason for the unclear risk of bias was that the 2 studies did not adequately report the methods for random sequence generation or allocation concealment. ${ }^{29,34}$ 
Table I Baseline characteristics of patients in the trials included in the meta-analysis

\begin{tabular}{|c|c|c|c|c|c|c|}
\hline Study & Country & Treatment regimen & $\begin{array}{l}\text { Patients } \\
\text { (n) }\end{array}$ & $\begin{array}{l}\text { Male/ } \\
\text { female }\end{array}$ & Age (y) & $\begin{array}{l}\text { Duration of current } \\
\text { episode }(m)\end{array}$ \\
\hline \multirow[t]{2}{*}{ Fava et $\mathrm{al}^{29}$} & USA & Adjunctive aripiprazole $2 \mathrm{mg} /$ day & 56 & $19 / 56$ & $45.36 \pm 10.35$ & NR \\
\hline & & Placebo & 169 & $61 / 169$ & $45.06 \pm 11.34$ & NR \\
\hline \multirow[t]{2}{*}{ Berman et $\mathrm{al}^{30}$} & USA & Adjunctive aripiprazole $2-20 \mathrm{mg} /$ day & 177 & $39 / 138$ & $45.1 \pm 10.6$ & $18.8(2.1-433.1)$ \\
\hline & & Placebo & 172 & $55 / 117$ & $45.6 \pm 11.3$ & $17.2(1.6-236.5)$ \\
\hline \multirow[t]{2}{*}{ Lin et $\mathrm{al}^{31}$} & China & Adjunctive aripiprazole $2.5 \mathrm{mg} /$ day & 21 & $17 / 4$ & $35.86 \pm|1.6|$ & NR \\
\hline & & Placebo & 20 & $16 / 4$ & $35.15 \pm 10.03$ & NR \\
\hline \multirow[t]{2}{*}{ Berman et $\mathrm{al}^{32}$} & USA & Adjunctive aripiprazole $2-15 \mathrm{mg} /$ day & 182 & $70 / 112$ & $46.5 \pm 10.6$ & $38.6 \pm 59$ \\
\hline & & Placebo & 176 & $63 / 113$ & $44.2 \pm 10.9$ & $43.6 \pm 53.8$ \\
\hline \multirow[t]{2}{*}{ Marcus et al ${ }^{33}$} & USA & Adjunctive aripiprazole $2-20 \mathrm{mg} /$ day & 191 & $65 / 126$ & $44.6 \pm 11.0$ & $43.7 \pm 68.0$ \\
\hline & & Placebo & 190 & $62 / 128$ & $44.4 \pm 10.7$ & $48.5 \pm 88.8$ \\
\hline \multirow[t]{2}{*}{ Lenze et $\mathrm{al}^{34}$} & USA & Adjunctive aripiprazole $10 \mathrm{mg} /$ day & 91 & $52 / 39$ & 66.4 & 27.5 \\
\hline & & Placebo & 90 & $51 / 39$ & 65.7 & 24.3 \\
\hline \multirow[t]{2}{*}{ Schüle et $\mathrm{a}^{35}$} & Germany & $15 \mathrm{mg} /$ day aripiprazole $+45 \mathrm{mg} /$ day mirtazapine & 20 & $9 / 11$ & $44.7 \pm 12.58$ & NR \\
\hline & & 45 mg/day mirtazapine & 20 & $4 / 16$ & $50.30 \pm 15.63$ & NR \\
\hline \multirow[t]{3}{*}{ Kamijima et $a^{36}$} & Japan & Adjunctive aripiprazole $3-15 \mathrm{mg} /$ day & 194 & $101 / 93$ & $38.1 \pm 9.6$ & $17.5 \pm 26.1$ \\
\hline & & Adjunctive aripiprazole $3 \mathrm{mg} /$ day & 197 & $124 / 73$ & $39.2 \pm 9.1$ & $15.7 \pm 21.6$ \\
\hline & & Placebo & 195 & $115 / 80$ & $38.7 \pm 9.2$ & $15.6 \pm 16.4$ \\
\hline
\end{tabular}

Note: Data presented as $\mathrm{n}$; mean $\pm \mathrm{SD}$; or mean (range).

Abbreviations: $m$, months; NR, not reported; SD, standard deviation; $y$, years.

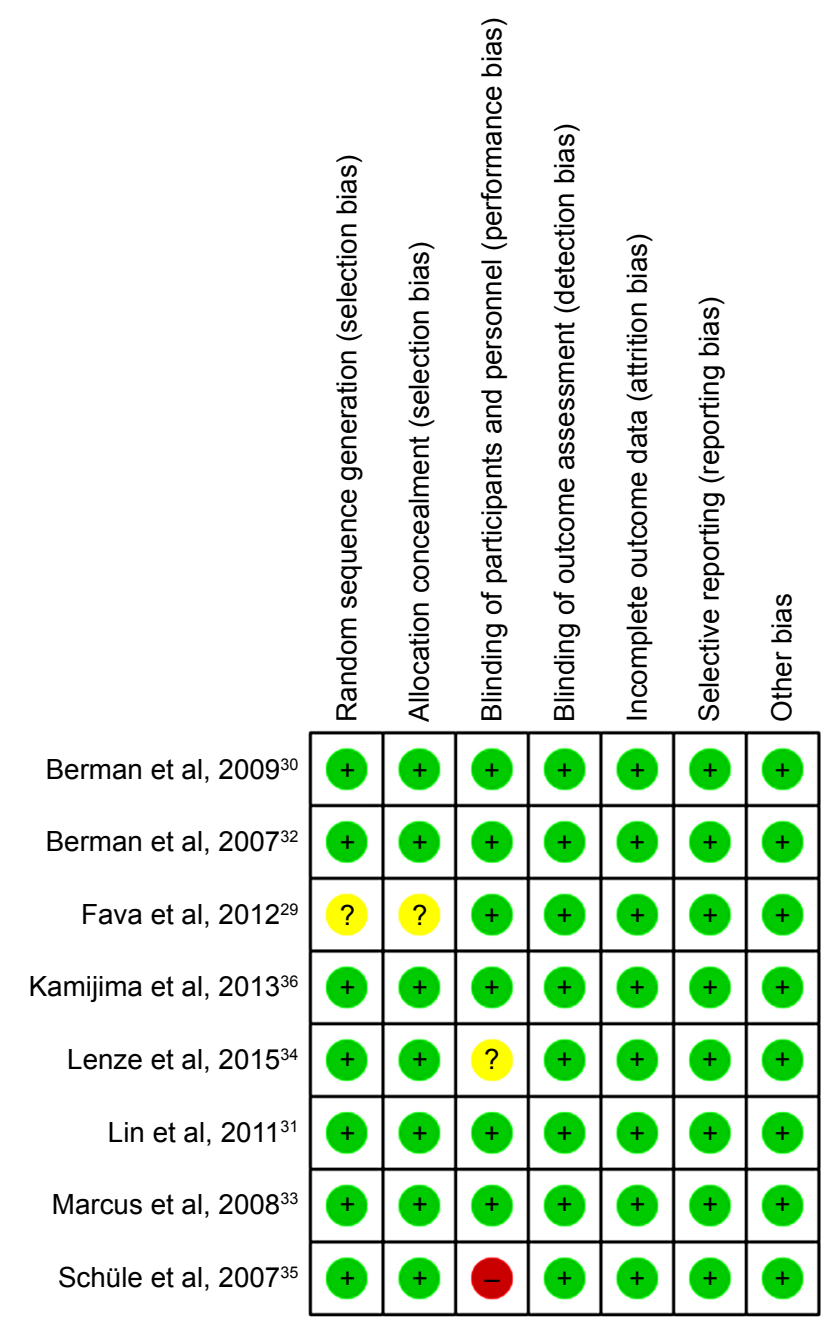

Figure 2 Risk of bias summary.
The GRADE evidence profiles for these outcomes are shown in Table 2 . The quality of evidence was high for remission rate, response rate, changes from baseline in MADRS, CGI-S, and CGI-I scores, discontinuation due to adverse events, and adverse events, and moderate for changes from baseline in HAM-D17, SDS, IDS-SR scores.

\section{Remission rate}

All the included studies reported the data of remission rate. ${ }^{29-36}$ The remission rate in the adjunctive aripiprazole group and control group was $29.8 \%$ and $18.1 \%$, respectively. The aggregated results suggested that adjunctive aripiprazole was associated with a significantly higher remission rate than the control ( $\mathrm{RR}=1.64,95 \% \mathrm{CI}$ : 1.42 to $1.89 ; P<0.001)$ (Figure 3). No evidence of heterogeneity was found among the included studies $\left(I^{2}=0.0 \%, P=0.995\right)$.

Subgroup analysis was conducted based on the control. The pooled results showed that adjunctive aripiprazole had a higher remission rate than placebo ( $\mathrm{RR}=1.64,95 \% \mathrm{CI}: 1.43$ to $1.90 ; P<0.001$ ), but a comparable remission rate with mirtazapine alone when it was combined with mirtazapine ( $\mathrm{RR}=1.25,95 \% \mathrm{CI}: 0.39$ to $3.99 ; P=0.706$ ) (Figure 3 ).

\section{Response rate}

Seven studies reported the data of response rate. ${ }^{29-33,35,36}$ The response rate in the adjunctive aripiprazole group and control group was $43.1 \%$ and $31.3 \%$, respectively. Pooled results showed that adjunctive aripiprazole group was associated 


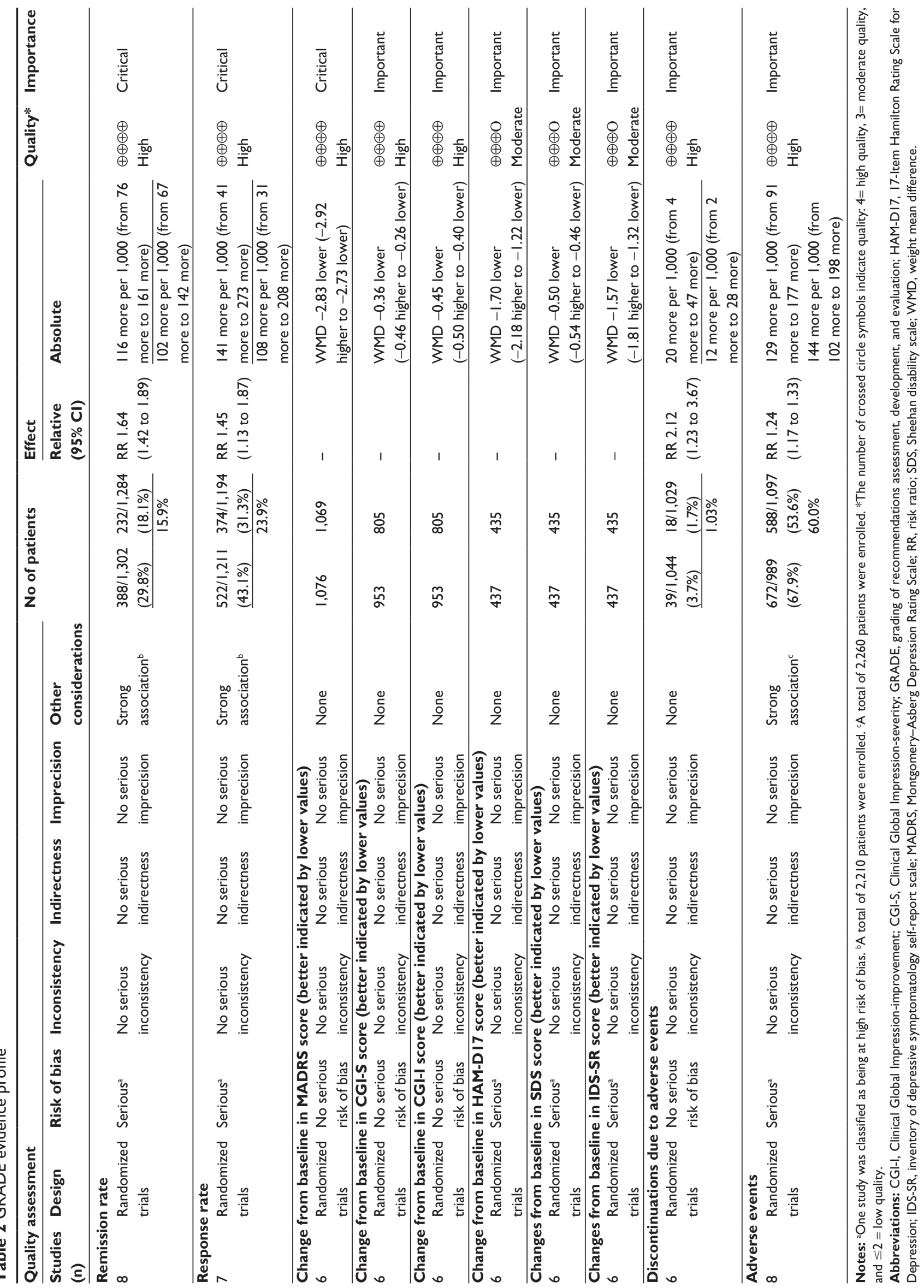




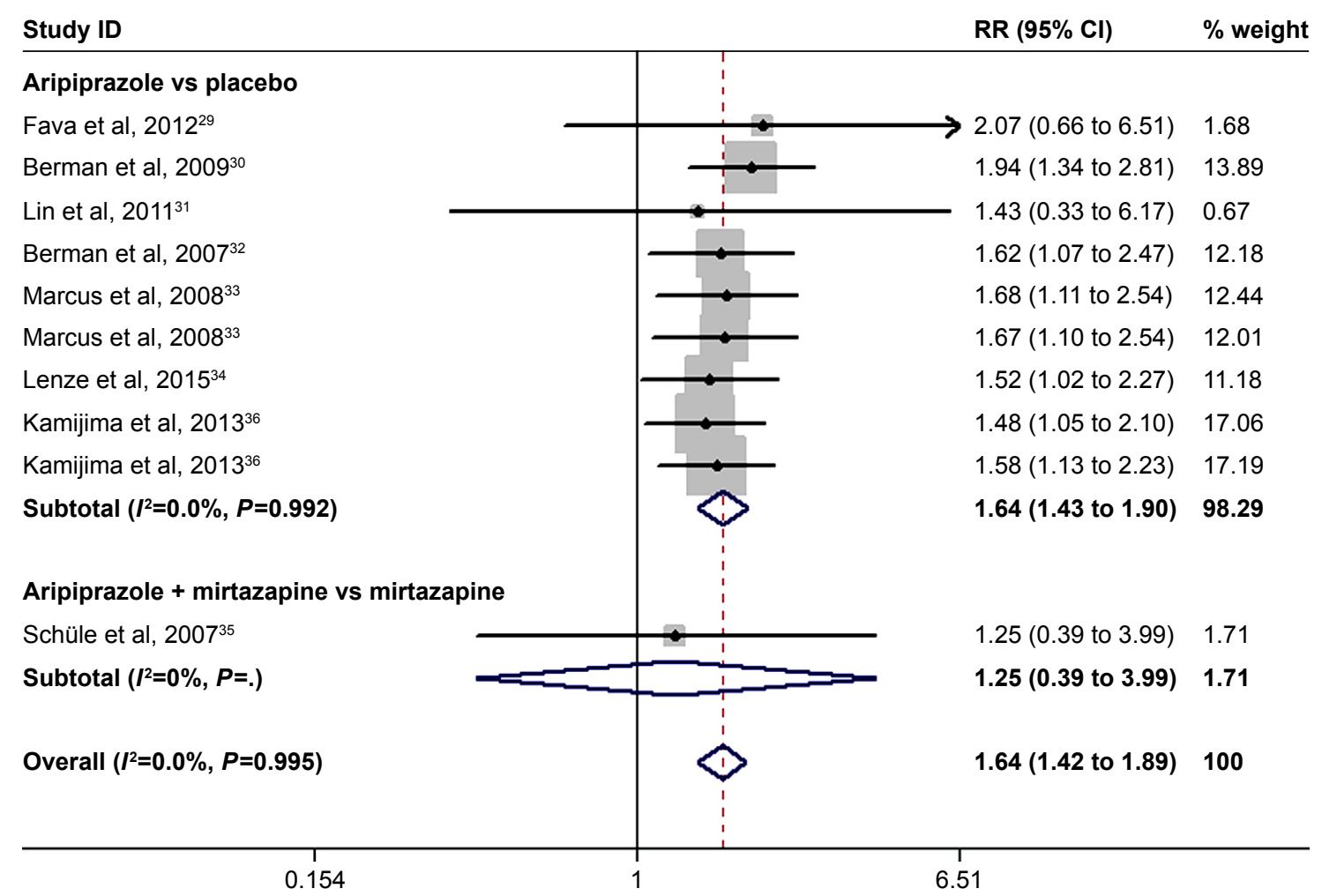

Figure 3 Forest plot showing the effect of adjunctive aripiprazole on remission rate. Abbreviations: $\mathrm{Cl}$, confidence interval; $\mathrm{RR}$, risk ratio.

with a significant greater response rate than other treatments ( $\mathrm{RR}=1.45,95 \% \mathrm{CI}: 1.13$ to $1.87 ; P=0.004$ ) (Figure 4). The test for heterogeneity was significant $\left(I^{2}=80.4 \%, P<0.001\right)$. Therefore, we conducted sensitivity to explore the potential source of heterogeneity. When we excluded the trial with the smallest sample size, ${ }^{31}$ the overall estimation changed slightly ( $\mathrm{RR}=1.46,95 \% \mathrm{CI}: 1.01$ to $2.12 ; P=0.032)$, and the heterogeneity was still present $\left(I^{2}=85.8 \%, P<0.001\right)$. When we excluded the study with outlier, ${ }^{35}$ the pooled result altered slightly ( $\mathrm{RR}=1.56,95 \% \mathrm{CI}: 1.01$ to $2.35 ; P=0.035)$, but the heterogeneity was still present $\left(I^{2}=86.3 \%, P<0.001\right)$. When we excluded the other studies individually, the overall combination and heterogeneity did not change substantially.

Subgroup analysis was conducted based on the control. The results showed that adjunctive aripiprazole had a higher response rate than placebo $(\mathrm{RR}=1.50,95 \% \mathrm{CI}$ : 1.14 to 1.98 ; $P=0.004)$, but a comparable response rate with mirtazapine alone when it was combined with mirtazapine $(\mathrm{RR}=1.09$, 95\% CI: 0.64 to $1.86 ; P=0.749$ ) (Figure 4).

\section{The change from baseline in MADRS score}

Six studies reported the data of changes from baseline in MADRS score. ${ }^{29,30,32-34,36}$ The pooled results suggested that the mean change in MADRS score was significantly greater in patients receiving adjunctive aripiprazole than in those treated with adjunctive placebo (WMD $=-2.83$, 95\% CI: -2.92 to $-2.73 ; P<0.001$ ) (Figure 5). There was no significant heterogeneity among the included studies $\left(I^{2}=0.0 \%, P=0.630\right)$.

\section{The changes from baseline in CGI-I and CGI-S scores}

Six studies reported the data of changes from baseline in CGI-S and CGI-I scores. ${ }^{29-33,36}$ The aggregated results of these studies demonstrated that adjunctive aripiprazole had greater decrease in CGI-S score (WMD $=-0.36,95 \% \mathrm{CI}:-0.46$ to $-0.26 ; P<0.001)$ and CGI-I score $(\mathrm{WMD}=-0.45,95 \% \mathrm{CI}:-0.50$ to $-0.40 ; P<0.001)$ than adjunctive placebo (Figure 6). There was significant heterogeneity among the included studies for the CGI-S score. Therefore, we conducted sensitivity analysis. When we excluded the trail conducted by Lin et $\mathrm{al}^{31}$ the overall estimation did not change substantially (WMD $=-0.44,95 \%$ CI: -0.51 to $-0.36 ; P<0.001)$, and the heterogeneity was still present $\left(I^{2}=97.1 \%, P<0.001\right)$. Further exclusion of any single study did not change the pooled estimation and heterogeneity substantially. 


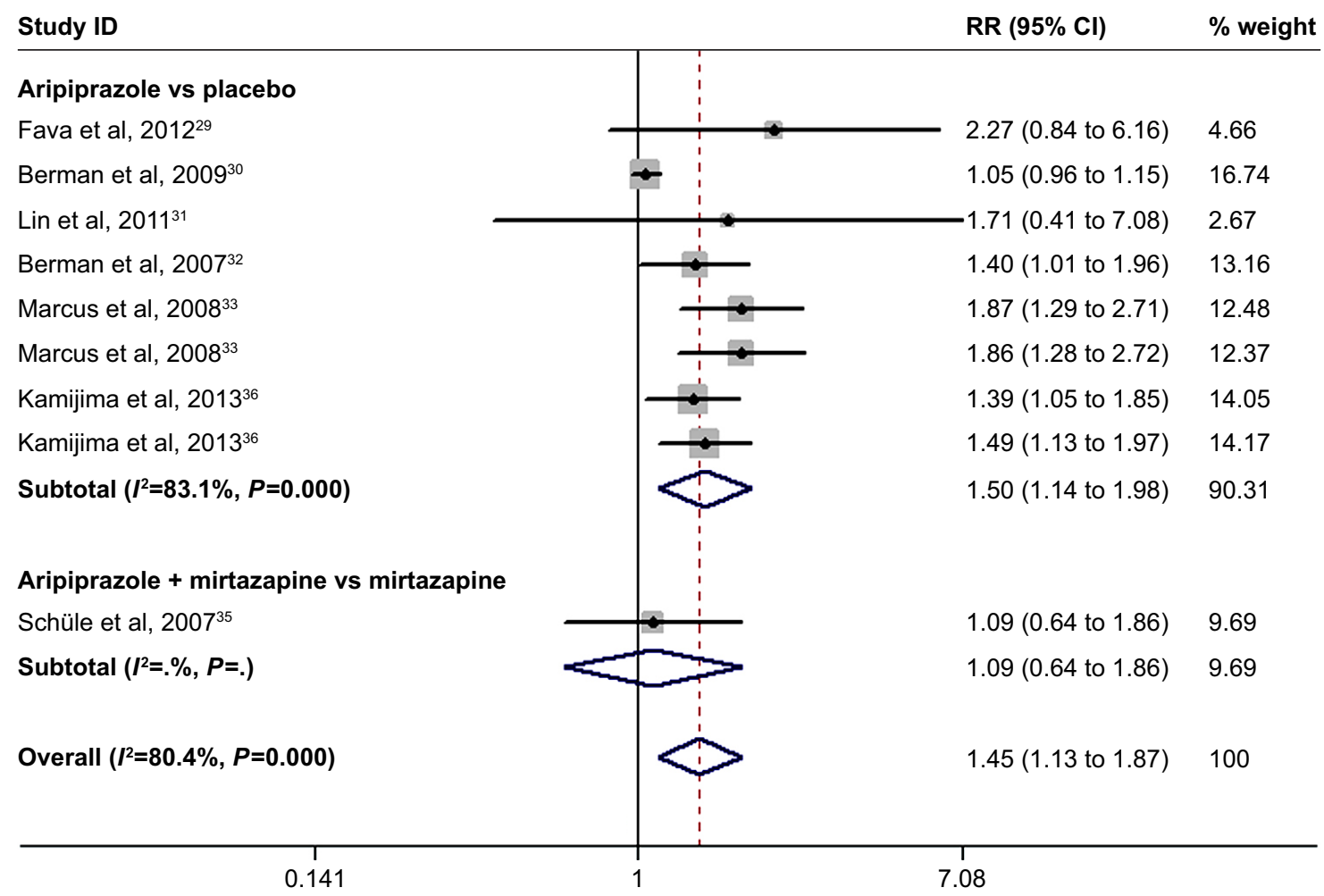

Figure 4 Forest plot showing the effect of adjunctive aripiprazole on response rate.

Note: Weights are from random effects analysis.

Abbreviations: $\mathrm{Cl}$, confidence interval; $\mathrm{RR}$, risk ratio.

The changes from baseline in HAM-DI7, SDS, and IDS-SR scores

Six studies reported the data of changes from baseline in HAM-D17, SDS, and IDS-SR. ${ }^{30-33,35,36}$ The pooled estimation showed that, compared with adjunctive placebo, adjunctive aripiprazole was associated with greater changes from baseline in HAM-D17 (WMD $=-1.70,95 \% \mathrm{CI}:-2.18$ to -1.22 ; $P<0.001$ ), SDS (WMD $=-0.50,95 \% \mathrm{CI}:-0.54$ to -0.46 ;

\begin{tabular}{|c|c|c|c|}
\hline Study ID & & WMD $(95 \% \mathrm{Cl})$ & $\%$ weigh \\
\hline Fava et al, $2012^{29}$ & $i$ & $-2.48(-4.79$ to -0.17$)$ & 0.18 \\
\hline Berman et al, $2009^{30}$ & 1 & $-3.70(-5.00$ to -2.40$)$ & 0.55 \\
\hline Berman et al, $2007^{32}$ & $\Rightarrow$ & $-3.00(-3.29$ to -2.71$)$ & 11.30 \\
\hline Marcus et al, $2008^{33}$ & 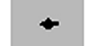 & $-2.80(-2.91$ to -2.69$)$ & 77.97 \\
\hline Lenze et al, $2015^{34}$ & $\rightarrow$ & $-2.80(-3.12$ to -2.48$)$ & 9.09 \\
\hline Kamijima et al, $2013^{36}$ & 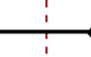 & $-2.20(-3.63$ to -0.77$)$ & 0.46 \\
\hline Kamijima et al, $2013^{36}$ & $\mathrm{i}$ & $-3.10(-4.53$ to -1.67$)$ & 0.46 \\
\hline Overall $\left(I^{2}=0.0 \%, P=0.630\right)$ & 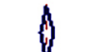 & $-2.83(-2.92$ to -2.73$)$ & 100 \\
\hline
\end{tabular}

Figure 5 Forest plot showing the effect of adjunctive aripiprazole on change from baseline in MADRS score. Abbreviations: $\mathrm{Cl}$, confidence interval; MADRS, Montgomery-Asberg Depression Rating Scale; WMD, weight mean difference. 


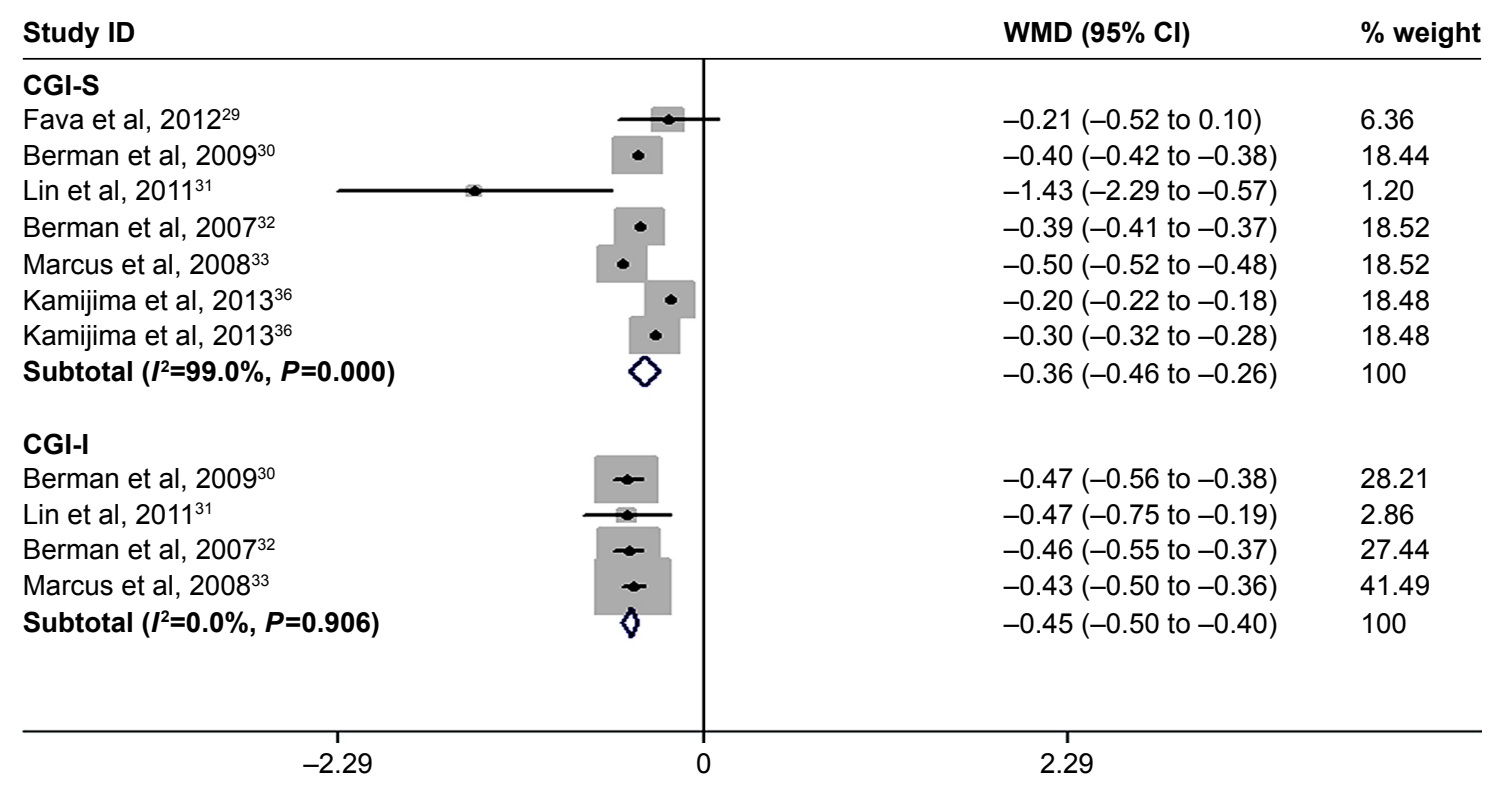

Figure 6 Forest plot showing the effect of adjunctive aripiprazole on changes from baseline in CGI-I and CGI-S scores. Note: Weights are from random effects analysis.

Abbreviations: CGI-I, Clinical Global Impression-improvement; CGI-S, Clinical Global Impression-severity; Cl, confidence interval; WMD, weight mean difference.

$P<0.001$ ), and IDS-SR scores (WMD $=-1.57,95 \% \mathrm{CI}:-1.81$ to $-1.32 ; P<0.001)$.

\section{Discontinuations due to adverse events}

Six studies reported the data of discontinuations due to adverse events. ${ }^{30-34,36}$ The rates of discontinuation due to adverse events in the adjunctive aripiprazole and adjunctive placebo groups were $3.7 \%$ and $1.7 \%$, respectively. Pooled estimation suggested that, adjunctive aripiprazole resulted in a significantly higher rate of discontinuation due to adverse events than placebo ( $\mathrm{RR}=2.12,95 \% \mathrm{CI}: 1.23$ to $3.67 ; P=0.007)$. The test for heterogeneity was not significant $\left(I^{2}=0.0 \%, P=0.810\right)$.

\section{Adverse events}

All the studies reported the data of adverse events. ${ }^{29-36}$ Overall, the incidence of adverse events in the adjunctive aripiprazole and adjunctive placebo groups was $67.9 \%$ and $53.6 \%$, respectively. The aggregated results showed that adjunctive aripiprazole had a significantly higher incidence of adverse events than the adjunctive placebo $(\mathrm{RR}=1.24$, 95\% CI: 1.17 to $1.33 ; P<0.001)$. The test for heterogeneity was not significant $\left(I^{2}=0.0 \%, P=0.699\right)$.

Compared with adjunctive placebo, adjunctive aripiprazole induced a significantly higher incidence of constipation $(\mathrm{RR}=2.33,95 \% \mathrm{CI}: 1.21$ to $4.50 ; P=0.011)$, fatigue $(\mathrm{RR}=1.68$, 95\% CI: 1.04 to $2.70 ; P=0.033)$, akathisia $(\mathrm{RR}=4.47,95 \% \mathrm{CI}$ : 1.77 to $11.28 ; P=0.002)$, insomnia $(\mathrm{RR}=2.19,95 \% \mathrm{CI}: 1.35$ to $3.54 ; P=0.001)$, restlessness ( $\mathrm{RR}=4.51,95 \% \mathrm{CI}: 2.36$ to 8.63; $P<0.001)$, and blurred vision ( $\mathrm{RR}=4.05,95 \% \mathrm{CI}: 1.68$ to $9.75 ; P=0.002$ ) (Table 3 ).

\section{Meta-regression analysis}

We first conducted univariate meta-regression analyses for each of the following variables: duration of follow-up, dosage of aripiprazole, sample size, and the control agent. The results showed that there was no significant association of effect size with these variables for the response rate (duration of follow-up, $P=0.258$; dosage of aripiprazole, $P=0.165$; sample size, $P=0.762$; control agent, $P=0.544$ ). This suggested that these variables were not significant and independent predictors for heterogeneity.

Table 3 Summary of the RRs with $95 \%$ Cls of adverse events

\begin{tabular}{|c|c|c|}
\hline Adverse events & $\operatorname{RR}(95 \% \mathrm{Cl})$ & P-value \\
\hline Constipation & 2.33 (I.2I to 4.49$)$ & 0.011 \\
\hline Diarrhea & 0.81 (0.49 to I.35) & 0.416 \\
\hline Dry mouth & $0.93(0.25$ to 3.49$)$ & 0.912 \\
\hline Nausea & 0.91 (0.60 to I.39) & 0.659 \\
\hline Fatigue & 1.68 (1.04 to 2.70$)$ & 0.033 \\
\hline Akathisia & 4.47 (I.77 to II.28) & 0.002 \\
\hline Headache & 0.81 (0.56 to I.I8) & 0.280 \\
\hline Somnolence & $\mathrm{I} .86(0.64$ to 5.43$)$ & 0.256 \\
\hline Insomnia & 2.19 (1.35 to 3.54$)$ & 0.001 \\
\hline Dizziness & $\mathrm{I} .45(0.50$ to 4.20$)$ & 0.492 \\
\hline Restlessness & 4.51 (2.36 to 8.63$)$ & $<0.001$ \\
\hline Blurred vision & 4.05 ( 1.68 to 9.75$)$ & 0.002 \\
\hline Tremor & $2.49(0.99$ to 6.28$)$ & 0.054 \\
\hline
\end{tabular}

Abbreviations: $\mathrm{Cl}$, confidence interval; $\mathrm{RR}$, risk ratio. 


\section{Publication bias}

We used the Egger's and Begg's tests to assess the publication bias, and the results showed that there was no evidence of publication bias (Egger's test: $t=-0.66, P=0.532$; Begg's test: $Z=0.62, P=0.536)$.

\section{Discussion}

The objective of this meta-analysis was to evaluate the efficacy, acceptability, safety, and quality of life of adjunctive aripiprazole in the treatment of patients with TRD. Our metaanalysis suggested that adjunctive aripiprazole was associated with a significantly higher remission rate and response rate than other treatments. In addition, adjunctive aripiprazole had greater changes in the scores of MADRS, CGI-S, CGI-I, HAM-D17, SDS, and IDS-SR. There were more patients treated with adjunctive aripiprazole who discontinued their studies due to adverse events, and more patients treated with adjunctive aripiprazole who experienced adverse events than those with other treatments. These results help to clarify the risk-benefit profiles of adjunctive aripiprazole for clinicians in the treatment of TRD patients.

There have been 2 published systematic review and metaanalysis of augmentation agents for TRD patients. ${ }^{37,38}$ In these studies, the authors evaluated the efficacy, acceptability, and tolerability of several augmentation agents for TRD patients, including aripiprazole, bupropion, buspirone, lamotrigine, lithium, methylphenidate, olanzapine, pindolol, quetiapine, risperidone, and thyroid hormone. Their results suggested that these antipsychotic augmentations have proven efficacious in reducing the depressive symptoms, and aripiprazole also showed benefits in improving the quality of life in TRD patients. ${ }^{37,38}$ Our study spends on the prior studies in providing more significant evidence for the use of adjunctive aripiprazole in TRD. First, the present meta-analysis had a more enlarged sample sizes than the previous analysis, which enhanced the statistical power to assess this effect. In this meta-analysis, we included 8 RCTs involving 2,260 patients, whereas in the previous 2 meta-analysis, there were only 4 RCTs of 1,317 patients focusing on the adjunctive aripiprazole. Second, we also conducted subgroup analysis based on control agent to evaluate the impact of these factors on the overall estimates, which was not analyzed in the previous meta-analysis..$^{37,38}$ Third, in this study, we also evaluated the effects of adjunctive aripiprazole on the changes from baseline in the scores of MADRS, CGI-S, CGI-I, HAM-D17, SDS, and IDS-SR, which were not performed in the previous meta-analysis..$^{37,38}$ The enlarged sample size has increased the statistical power to provide more reliable effect estimates, and the additional analysis provided more comprehensive information for the clinical physicians.

In this meta-analysis, we found that the adjunctive aripiprazole was associated with a significantly higher remission rate and response rate than the control. These findings were consistent with results of the previous controlled studies. ${ }^{37,38}$ In the double-blind, placebo-controlled study of Berman $\mathrm{RM},{ }^{30} 349$ patients with inadequate response were randomly assigned to the adjunctive aripiprazole ( $\mathrm{n}=177,20 \mathrm{mg} /$ day) group or adjunctive placebo $(\mathrm{n}=172)$ group..$^{30}$ At 14 weeks, the remission rates in the 2 groups were $36.8 \%$ and $18.9 \%$, and response rates were $87 \%$ and $83 \%$, respectively. ${ }^{30}$ This indicated that adjunctive aripiprazole exhibited significantly better efficacy than placebo in the remission rate and response rate. Similarly, in another randomized, doubleblind, placebo-controlled trial, ${ }^{33}$ the authors also reported superior effects of adjunctive aripiprazole over placebo..$^{33}$ In that study, 381 patients were randomized to adjunctive aripiprazole $(\mathrm{n}=191$, starting dose $5 \mathrm{mg} /$ day, dose adjustments $2-20 \mathrm{mg}$ /day, mean endpoint dose of $11.0 \mathrm{mg} /$ day) or adjunctive placebo $(n=190) .{ }^{33}$ They were treated with these adjunctive agents for 8 weeks. At the endpoint, patients who received the adjunctive aripiprazole had significantly greater remission rate $(25.4 \%$ vs $15.2 \%)$ and response rate $(32.4 \%$ vs $17.4 \%$ ) than those treated with adjunctive placebo. ${ }^{33}$

In contrast to their positive results, Lin et al reported a comparable effect of adjunctive aripiprazole with placebo. ${ }^{31}$ In that study, 21 and 20 patients were assigned to the aripiprazole group and placebo group, respectively. ${ }^{31}$ They received $2.5 \mathrm{mg}$ /day aripiprazole for 10 weeks..$^{31}$ At the endpoint, the remission rate and response rate in the aripiprazole group were $71.4 \%$ and $85.7 \%$, compared with $50 \%$ and $50 \%$ in the placebo group, respectively. ${ }^{31}$ However, the differences between them were not significant. The authors attributed the negative results to the small sample size. At 4 weeks, the remission rate and response rate were significantly different, but these benefit effects were not observed at the 6 weeks because of the high dropout rate. ${ }^{31}$

According to this study, the change from baseline in MADRS score was significantly greater in the adjunctive aripiprazole group than that in the placebo group. This result was inconsistent with reports of the previous studies. ${ }^{30,31}$ Kamijima et $\mathrm{al}^{36}$ conducted a randomized, double-blind, placebocontrolled study (ADMIRE study), which assessed the efficacy and safety of a fixed dose ( $3 \mathrm{mg} /$ day) and flexible dose (3-15 mg/day) schedule of adjunctive aripiprazole in Japanese patients. ${ }^{36}$ In that study, 286 patients were randomly assigned to the adjunctive treatment with flexible-dose aripiprazole 
group ( $\mathrm{n}=194)$, fixed-dose aripiprazole group $(\mathrm{n}=197)$, and placebo group $(n=195) \cdot{ }^{36}$ After the 8 week treatment, patients who received either the adjunctive fixed dose or flexible dose of aripiprazole achieved a significantly greater reduction from baseline in the MADRS score ( -10.5 and -9.6 , respectively) than those treated with adjunctive placebo $(-7.4){ }^{36}$

Whereas in another double-blind, placebo-controlled study conducted by Fava et al, ${ }^{29}$ they also found a greater improvement of adjunctive aripiprazole in MADRS score, but this change was not significantly different when compared with the placebo. ${ }^{29}$ In that study, 255 patients were randomized to adjunctive treatment with aripiprazole $2 \mathrm{mg}$ /day or placebo, with a 2:3:3 randomization ratio to drug/drug (aripiprazole $2 \mathrm{mg} /$ day in Phase I; $5 \mathrm{mg}$ /day in Phase II), placebo/placebo (placebo in both phases), and placebo/drug (placebo in Phase I; aripiprazole $2 \mathrm{mg}$ /day in Phase II). ${ }^{29}$ The MARDS mean change for aripiprazole $2 \mathrm{mg}$ /day decreased by 8.5 points in Phase I and 5.8 points in Phase II, compared with 8.1 points in Phase I and 3.3 points in Phase II for placebo, respectively. ${ }^{36}$ The difference between the 2 groups was not significant (weighted difference $=-1.51, P=0.0649$ ). ${ }^{36}$ When the data were pooled from both phases, the mean MADRS score of adjunctive aripiprazole was 1.51 points more than placebo; however, the difference was still not statistically significant $(P=0.0649) .{ }^{36}$ The authors thought that the interpretation for these negative results were complex. Since the placebo response rate in Phase I was in line with that in the previous positive studies, the robust effects of adjunctive aripiprazole $2-15 \mathrm{mg}$ /day in other trials suggested that the efficacy of low-dose aripiprazole $2 \mathrm{mg}$ /day was minimal. ${ }^{36}$ Despite the dosage of aripiprazole was raised to $5 \mathrm{mg}$ /day in the second phase, the aripiprazole-placebo difference was only $4.3 \%$, suggesting that the low-dose aripiprazole might not be efficacious, and the $5 \mathrm{mg}$ /day aripiprazole only presented the minimal-effect benefits. ${ }^{36}$

With regard to the safety profile, our results suggested that patients treated with adjunctive aripiprazole experienced a significantly higher incidence of adverse events. Moreover, there were more patients treated in the adjunctive aripiprazole group who discontinued the study due to adverse events than those in the placebo group. The most commonly occurring adverse events with aripiprazole included akathisia, constipation, fatigue, insomnia, restlessness, blurred vision, diarrhea, nausea, and fatigue. Most of these adverse events were generally mild-to-moderate in severity. Akathisia was the most common adverse event with adjunctive aripiprazole. In this study, adjunctive aripiprazole was associated with a 3.47-fold greater likelihood of akathisia than the placebo ( $\mathrm{RR}=4.47,95 \% \mathrm{CI}: 1.77$ to $11.28 ; P=0.002)$. This result was consistent with the finding of the study conducted by Lenze et al. ${ }^{34}$ In that study, there was a significantly higher rate of akathisia with adjunctive aripiprazole than with placebo $(26.7 \%$ vs $12.2 \%) .{ }^{34}$ Moreover, akathisia resulted in a temporary increase in suicidal thoughts in 3 patients treated with aripiprazole and trial discontinuation in another 1 patient. ${ }^{34}$ Thus, health care professionals should be aware of these adverse effects of aripiprazole and adjust dose or potentially switch treatment.

There were several limitations in this meta-analysis. First, our analysis was based on 8 RCTs, and some of them had a relatively small sample size $(\mathrm{n}<100)$. Overestimation of the treatment effect is more likely in smaller trials when compared with larger trials. Second, some of the subgroup analysis was based only on 3-4 studies; thus, conclusion about the remission rate and response rate of adjunctive aripiprazole should be interpreted with caution. Third, we found considerable heterogeneity across the studies in our meta-analysis. It was not surprising given the differences in the study population, duration of the treatment, dosage of aripiprazole, and the definitions of TRD and response. These factors account for the heterogeneity and could affect our results. Fourth, it should be noted that all the included trials were sponsored by pharmaceutical companies; thus, we could not rule out the existence of possible bias that was brought by the inherent conflict of interest.

\section{Conclusion}

The present meta-analysis suggested that adjunctive aripiprazole significantly exhibited benefit effects in improving the response rate, remission rate, and the quality of life in patients with TRD. However, clinicians should interpret these findings cautiously in light of the evidence of potential treatmentrelated side effects. More large-scale, well-designed RCTs are needed to verify our findings.

\section{Disclosure}

The authors report no conflicts of interest in this work.

\section{References}

1. Whiteford HA, Degenhardt L, Rehm J, et al. Global burden of disease attributable to mental and substance use disorders: findings from the Global Burden of Disease Study 2010. Lancet. 2013;382(9904): 1575-1586.

2. Callahan CM, Kroenke K, Counsell SR, et al; IMPACT Investigators. Treatment of depression improves physical functioning in older adults. J Am Geriatr Soc. 2005;53(3):367-373.

3. Gallo JJ, Morales KH, Bogner HR, et al. Long term effect of depression care management on mortality in older adults: follow-up of cluster randomized clinical trial in primary care. BMJ. 2013;346:f2570.

4. Fava M. Diagnosis and definition of treatment-resistant depression. Biol Psychiatry. 2003;53(8):649-659. 
5. Trivedi MH, Rush AJ, Wisniewski SR, et al; STAR*D Study Team. Evaluation of outcomes with citalopram for depression using measurement-based care in STAR*D: implications for clinical practice. Am J Psychiatry. 2006;163(1):28-40.

6. Nemeroff CB. The burden of severe depression: a review of diagnostic challenges and treatment alternatives. J Psychiatr Res. 2007; 41(3-4):189-206.

7. Sackeim HA. The definition and meaning of treatment-resistant depression. J Clin Psychiatry. 2001;62 (Suppl 16):10-17.

8. Fava M, Rush AJ. Current status of augmentation and combination treatments for major depressive disorder: a literature review and a proposal for a novel approach to improve practice. Psychother Psychosom. 2006;75(3):139-153.

9. Maust DT, Oslin DW, Thase ME. Going beyond antidepressant monotherapy for incomplete response in nonpsychotic late-life depression: a critical review. Am J Geriatr Psychiatry. 2013;21(10):973-986.

10. Cooper C, Katona C, Lyketsos K, et al. A systematic review of treatments for refractory depression in older people. Am J Psychiatry. 2011;168(7):681-688.

11. Lam RW, Kennedy SH, Grigoriadis S, et al; Canadian Network for Mood and Anxiety Treatments (CANMAT). Canadian Network for Mood and Anxiety Treatments (CANMAT) clinical guidelines for the management of major depressive disorder in adults. III. Pharmacotherapy. J Affect Disord. 2009;117 (Suppl 1):S26-S43.

12. Fleurence R, Williamson R, Jing Y, et al. A systematic review of augmentation strategies for patients with major depressive disorder. Psychopharmacol Bull. 2009;42(3):57-90.

13. Burris KD, Molski TF, Xu C, et al. Aripiprazole, a novel antipsychotic, is a high-affinity partial agonist at human dopamine D2 receptors. $J$ Pharmacol Exp Ther. 2002;302(1):381-389.

14. Jordan S, Koprivica V, Chen R, Tottori K, Kikuchi T, Altar CA. The antipsychotic aripiprazole is a potent, partial agonist at the human 5-HT1A receptor. Eur J Pharmacol. 2002;441(3):137-140.

15. Shapiro DA, Renock S, Arrington E, et al. Aripiprazole, a novel atypical antipsychotic drug with a unique and robust pharmacology. Neuropsychopharmacology. 2003;28(8):1400-1411.

16. Jon DI, Kim DH, Seo HJ, et al. Augmentation of aripiprazole for depressed patients with an inadequate response to antidepressant treatment: a 6-week prospective, open-label, multicenter study. Clin Neuropharmacol. 2013;36(5):157-161.

17. Moher D, Liberati A, Tetzlaff J, Altman DG. Preferred reporting items for systematic reviews and meta-analyses: the PRISMA statement. BMJ. 2009;339:b2535.

18. Higgins JP, Altman DG, Gotzsche PC, et al; Cochrane Statistical Methods Group. The cochrane collaboration's tool for assessing risk of bias in randomised trials. BMJ. 2011;343:d5928.

19. Guyatt GH, Oxman AD, Vist GE, et al; GRADE Working Group. GRADE: an emerging consensus on rating quality of evidence and strength of recommendations. BMJ. 2008;336(7650):924-926.

20. Higgins JP, Thompson SG, Deeks JJ, Altman DG. Measuring inconsistency in meta-analyses. BMJ. 2003;327(7414):557-560.

21. DerSimonian R, Laird N. Meta-analysis in clinical trials. Control Clin Trials. 1986;7(3):177-188.

22. Mantel N, Haenszel W. Statistical aspects of the analysis of data from retrospective studies of disease. J Natl Cancer Inst. 1959;22(4):719-748.

23. Begg CB, Mazumdar M. Operating characteristics of a rank correlation test for publication bias. Biometrics. 1994;50(4):1088-1101.
24. Egger M, Davey Smith G, Schneider M, Minder C. Bias in meta-analysis detected by a simple, graphical test. BMJ. 1997;315(7109):629-634.

25. Fabrazzo M, Perris F, Monteleone P, Esposito G, Catapano F, Maj M. Aripiprazole augmentation strategy in clomipramine-resistant depressive patients: an open preliminary study. Eur Neuropsychopharmacol. 2012;22(2):132-136.

26. Naylor JC, Kilts JD, Bradford DW, et al. A pilot randomized placebocontrolled trial of adjunctive aripiprazole for chronic PTSD in US military Veterans resistant to antidepressant treatment. Int Clin Psychopharmacol. 2015;30(3):167-174.

27. Kaneriya SH, Robbins-Welty GA, Smagula SF, et al. Predictors and moderators of remission with aripiprazole augmentation in treatmentresistant late-life depression: an analysis of the IRL-GRey Randomized Clinical Trial. JAMA Psychiatry. 2016;73(4):329-336.

28. Yoshimura R, Kishi T, Hori H, et al. Comparison of the efficacy between paroxetine and sertraline augmented with aripiprazole in patients with refractory major depressive disorder. Progress Neuropsychopharmacol Biol Psychiatry. 2012;39(2):355-357.

29. Fava M, Mischoulon D, Iosifescu D, etal. A double-blind, placebo-controlled study of aripiprazole adjunctive to antidepressant therapy among depressed outpatients with inadequate response to prior antidepressant therapy (ADAPT-A Study). Psychother Psychosom. 2012;81(2):87-97.

30. Berman RM, Fava M, Thase ME, et al. Aripiprazole augmentation in major depressive disorder: a double-blind, placebo-controlled study in patients with inadequate response to antidepressants. CNS Spectr. 2009;14(4):197-206.

31. Lin $\mathrm{CH}$, Lin SH, Jang FL. Adjunctive low-dose aripiprazole with standard-dose sertraline in treating fresh major depressive disorder: a randomized, double-blind, controlled study. JClin Psychopharmacol. 2011;31(5):563-568.

32. Berman RM, Marcus RN, Swanink R, et al. The efficacy and safety of aripiprazole as adjunctive therapy in major depressive disorder: a multicenter, randomized, double-blind, placebo-controlled study. J Clin Psychiatry. 2007;68(6):843-853.

33. Marcus RN, McQuade RD, Carson WH, et al. The efficacy and safety of aripiprazole as adjunctive therapy in major depressive disorder: a second multicenter, randomized, double-blind, placebo-controlled study. J Clin Psychopharmacol. 2008;28(2):156-165.

34. Lenze EJ, Mulsant BH, Blumberger DM, et al. Efficacy, safety, and tolerability of augmentation pharmacotherapy with aripiprazole for treatment-resistant depression in late life: a randomised, double-blind, placebo-controlled trial. Lancet. 2015;386(10011):2404-2412.

35. Schüle C, Baghai TC, Eser D, et al. Mirtazapine monotherapy versus combination therapy with mirtazapine and aripiprazole in depressed patients without psychotic features: a 4-week open-label parallel-group study. World J Biol Psychiatry. 2007;8(2):112-122.

36. Kamijima K, Higuchi T, Ishigooka J, et al; ADMIRE StudyGroup. Aripiprazole augmentation to antidepressant therapy in Japanese patients with major depressive disorder: a randomized, double-blind, placebo-controlled study (ADMIRE study). J Affect Disord. 2013;151(3):899-905.

37. Zhou X, Ravindran AV, Qin B, et al. Comparative efficacy, acceptability, and tolerability of augmentation agents in treatment-resistant depression: systematic review and network meta-analysis. $J$ Clin Psychiatry. 2015;76(4):e487-e498.

38. Zhou X, Keitner GI, Qin B, et al. Atypical antipsychotic augmentation for treatment-resistant depression: a systematic review and network meta-analysis. Int J Neuropsychopharmacol. 2015;18(11):pyv060.
Neuropsychiatric Disease and Treatment

\section{Publish your work in this journal}

Neuropsychiatric Disease and Treatment is an international, peerreviewed journal of clinical therapeutics and pharmacology focusing on concise rapid reporting of clinical or pre-clinical studies on a range of neuropsychiatric and neurological disorders. This journa is indexed on PubMed Central, the 'PsycINFO' database and CAS,

\section{Dovepress}

and is the official journal of The International Neuropsychiatric Association (INA). The manuscript management system is completely online and includes a very quick and fair peer-review system, which is all easy to use. Visit http://www.dovepress.com/testimonials.php to read real quotes from published authors. 